\title{
Chancen der Regionalen Ärztlichen Dienste der Invalidenversicherung
}

\author{
P. Winzeler
}

Momentan sind die Invalidenversicherung (IV) und der IV-ärztliche Dienst föderalistisch organisiert. Jeder Kanton hat eine eigene IV-Stelle und eigene IV-Ärztinnen/-Ärzte.

Mit der 4. Revision der Invalidenversicherung, die ab 1. Januar 2004 in Kraft tritt, wird der IV-ärztliche Dienst regionalisiert. Verteilt über die Schweiz werden etwa 10 Regionale Ärztliche Dienste (RAD) gebildet. Ein RAD erbringt somit versicherungsmedizinische Dienstleistungen für mehrere kantonale IV-Stellen. Das System der RAD wurde ab 2000 versuchsweise in vier RADPilotregionen geprüft.

Die interessante Frage ist nun: Was bringen die RAD? Um dies zu beantworten, möchte ich vier Themenbereiche diskutieren:

- Steigerung der Beurteilungsqualität;

- Harmonisierung der IV-ärztlichen Entscheide;

- Senkung der Anzahl Begutachtungen;

- Senkung der Rentenzahlen/Rentenkosten.

\section{Steigerung der Beurteilungsqualität}

Dies kann meines Erachtens klar bejaht werden.

Mit der Einführung der RAD kann der Personaletat der IV-Ärztinnen/-Ärzte aufgestockt werden. Die Regionalisierung erlaubt bzw. fordert das Anstellen von Spezialärztinnen/-ärzten der Fachbereiche Allgemeinmedizin / Innere Medizin, Rheumatologie/Orthopädie, Psychiatrie und Pädiatrie. Neben diesen zusätzlichen Spezialisten ist auch gefordert, dass die IV-Stellen beinahe alle Dossiers dem RAD zur Beurteilung vorlegen.

Voraussetzung der Qualitätssteigerung ist selbstverständlich, dass RAD-Ärztinnen/-Ärzte IV-rechtlich ausgebildet und à jour sind.

\section{Harmonisierung \\ der IV-ärztlichen Entscheide}

Korrespondenz: Dr. med. Peter Winzeler Sozialversicherungsanstalt des Kantons St. Gallen Brauerstrasse 54 CH-9016 St. Gallen
Auch diese Frage kann meines Erachtens klar bejaht werden.

Integraler Teil des RAD-Konzeptes ist eine kontinuierliche interne Fortbildung bezüglich IV-rechtlicher Beurteilungen. Wichtig ist zusätz- lich, dass benachbarte RAD Kontakt untereinander haben und gewisse Fragen in einem überregionalen Rahmen lösen.

Voraussetzung für eine Qualitätssteigerung durch Harmonisierung der IV-ärztlichen Entscheide ist auch hier, dass der Einführung von neuen Kolleginnen und Kollegen die notwendige Beachtung geschenkt und die Fortbildung ernstgenommen wird.

\section{Senkung der Anzahl Begutachtungen}

Mit dem Einführen der RAD werden IV-Ärztinnen/-Ärzte berechtigt, versicherte Personen selbst zu untersuchen, dies ähnlich zu den kreisärztlichen Untersuchungen bei der SUVA. Wie stark solche Untersuchungen die Anzahl der Begutachtungen senkt, ist schwer abzuschätzen.

IV-ärztliche Untersuchungen können bei unklarem Anspruch auf Renten oder berufliche Massnahmen sicher weiterhelfen. Bestimmt lassen sich auch gewisse Gesundheitsschäden klarer beurteilen. Hingegen glaube ich eher nicht, dass sich in komplexen Fällen mit IVärztlichen Untersuchungen polydisziplinäre und MEDAS-Begutachtungen ersetzen lassen (MEDAS = medizinische Abklärungsstellen des Bundes).

Offen ist natürlich auch, wie die Sozialgerichte IV-ärztliche Untersuchungen gewichten und wie stark sich die Gerichte auf solche Untersuchungen abstützen. Erfreulicherweise sind die Erfahrungen von den Pilot-RAD diesbezüglich positiv.

\section{Senkung der Rentenzahlen/ Rentenkosten}

Diese Frage beschäftigt uns alle und sie ist neben den Gesundheitskosten ein Politikum erster Güte.

Trotzdem bin ich skeptisch und dies aus dem einfachen Grund, weil die Anzahl der Rentenbezüger, bzw. die Rentenkosten, nur teilweise von medizinischen Faktoren und den Beurteilungen des RAD abhängen. Dazu zwei Beispiele: 
- Rentenanmeldungen und Gesundheitsschäden hängen sehr stark von der Wirtschaftslage ab. Je mehr die Wirtschaft ökonomisch optimiert wird, desto mehr fallen Personen mit gesundheitlichen Einschränkungen durch die Maschen und desto weniger werden Nischenarbeitsplätze für Handicapierte angeboten. Der Slogan «Arbeitslosigkeit macht krank» ist nicht von der Hand zu weisen.

- Rentenzusprachen hängen aber auch von juristischen Bemühungen der Rechtsanwältinnen/Rechtsanwälte und den Beurteilungen der Sozialgerichte ab. Das Durchsetzen von zusätzlichen Abklärungen und Begutachtungen verzögert die Entscheide der IV so stark, dass auch kleinere gesundheitliche Schäden eine grosse Chance haben, sich auszuweiten, therapieresistent und chronifiziert zu werden - Stichwort: Administrative Chronifizierung.

Das Resultat beider Beispiele ist ein «ausgewiesenes Anrecht auf eine Rente», unabhängig wie kompetent und effizient ein RAD arbeitet.

Zusammenfassend lässt sich feststellen, dass die Regionalen Ärztlichen Dienste sicher eine Qualitätsverbesserung der IV-Entscheide bringen werden, sie werden jedoch nicht alle Probleme der IV lösen können.

\title{
Menschenkonservierung
}

\author{
E. Danieli
}

Korrespondenz:

Dr. med. Enrico Danieli Seefeldstrasse 128 CH-8008 Zürich
Ich hatte die Anmeldung zur eintägigen Fortbildungsveranstaltung hinausgezögert, gewartet bis zuletzt. In reisserischer Aufmachung war auf eine neue, die zukünftige Lehre der Pathologie und damit die gesamte Medizin revolutionierende Konservierungstechnik von Kranken hingewiesen worden, und dass es eben dank dieser Technik nun gelinge, nicht mehr nach Krankheiten, sondern nach Kranken und ihren Pathologien anschaulich systematisch $\mathrm{zu}$ suchen. Kaum mag eine Rolle gespielt haben, dass ich nur wenige Tage zuvor von einem Kongress von Konservatoren gelesen und dabei erfahren hatte, dass bei diesem Kongress von neuartigen Verfahren zum Präparieren des Lebens die Rede gewesen war. Dabei hatte ich mich in der Lektüre des Zeitungsartikels verheddert, ich las nämlich von Enthäutungs- und Entfleischungsmethoden, von Skelettmontagen, aber auch von dank neuer Technik endgültig überwundenen Schäden in Präparatesammlungen, vom Präparationshilfsmittel Gefriertrockner, von der Klimatisierung von Ganzsammlungsgut, von dermoplastischen, sensationellen Lebendbefunden und von Leichengefrierfächern. Und es war mir bei der Lektüre vorgekommen, als würde die grossangekündigte Fortbildung nichts anderem dienen als der Weiterführung des gleichen Themas aus nun medizinischer Sicht: Mensch und Tier, so meine Gedanken, sind ununterscheidbar:
Glasaugensammlungen und Druckknopfaugen, Damhirschkalb- und Embryopräparat, Präparatoren für Fisch, Vogel, Mensch, Pilz, Insekt. Zum Gehen verleitet hatten mich dann eher der Tagungsort - hoch über der Stadt - und das Seminarhotel - exklusiv schon der Name: Grand Hotel - und sicher weniger das Thema: Die Lebendkonservierung. Revolution oder Theater?

Ich ging mit sehr gemischten Gefühlen zur Station der roten Standseilbahn, denn ob ich es wollte oder nicht, ich war plötzlich der festen Überzeugung, nun alles über die menschliche Konservierung oder besser: von der Checkliste für das menschliche Präparatorium zu erfahren. Glücklicherweise lenkte mich die Fahrt aufwärts im rumpelnden Wagen ab, fahl schien eine gelbe Wintersonne, Rauhreif verzauberte die Tannenäste, Kinderschlitten mit bimmelnden Glöcklein versperrten die Schiebetüren, diese Umgebung, dachte ich, verspricht professionelle Weiterbildung.

Und so enttäuschte das Hotel denn auch nicht, wurden doch schon beim Empfang Getränke herumgereicht, eilten Livrierte, mit Lachsbrötchen gefüllte Silberplatten auf ihren weiss behandschuhten Händen jonglierend, von Gruppe zu Gruppe, fragten schwarzgekleidete Damen nach Erfrischungswünschen, und auch die vor ihren Buden patrouillierenden Pharmavertreter lächelten einnehmend, beinahe ver- 
schwörerisch - wussten sie denn schon, was uns erwartete? - oder hintersinnig. Der Andrang war riesig, Kollegen von nah und fern waren angereist, es war also doch so, dass Sensationelles auf uns zukam. Und der im Hochparterre gelegene Jugendstilsaal sprengte alle Erwartungen: goldenverzierte Deckenstukkaturen, plüschbezogene Wände, Kronleuchter, wattiertes Gehen der Kellner, gedämpfte Gespräche der Mediziner hinter vorgehaltenen Händen. Nicht verwunderlich, dass meine Augen nun Mühe hatten, die Schrift auf der Leinwand zu entziffern, meine Ohren den Wörtern - Konservierungsmittel, Farbumschläge, Mazerierregeln, Styroporfüllungen und so weiter - kaum folgen konnten, mein Kopf die vorgeführten und ausführlich erläuterten Zusammenhänge zwischen, wie referiert wurde, tierischem Präparat und menschlichem Fall in keine direkte Beziehung zu bringen vermochte, alles Dargebotene und auch Dargestellte, also die auf die Leinwand anschaulich projizierten Tier- oder Menschenpräparate, die sogenannten «top shots» in Grossbuchstaben auf der Schreibtafel, die «key messages» auf «flyers», die «take home messages» auf den «hand outs», sich fremd anhörte, vielleicht waren meine Sinne einfach zu sehr gefangengenommen von den Flügeltüren, vom glänzenden
Riemenparkett, von der beigen, den Saal umrundenden Täfelung, von den schon in Nischen wartenden Hoteldienern.

Vor dem Ende der Tagung, ich hatte kaum mehr zugehört, mit geschlossenen Augen hatte ich die Schlussworte abgewartet, diese wie sonst nichts als Erlösung herbeisehnend, wurde nicht nur auf die ausschliesslich für den heutigen Tag in Vitrinen gelagerten und vor dem Vortragssaal nun ausgestellten menschlichen Präparatteile als bestens geeignetes Anschauungsmaterial für Lehre und Studium hingewiesen, sondern es wurden auch Spezialführungen zu den Menschengrosspräparaten angeboten, die bis heute in Klinikkellern verstaubt und als besondere Überraschung zum ersten Mal einer kleinen, auserlesenen Anzahl von Medizinern, natürlich unter strikter Geheimhaltung, zugänglich gemacht würden, es seien alles Trophäen, die meisten von Headhuntern auf der Flucht oder sonstwie um das Leben gebracht, nun gesammelt, lebendfrisch vom anatomisch-pathologischen Präparator zugeliefert, mit Hilfe der neuartigen Technik konserviert. Die Führungen, wie nicht anders zu erwarten, hatten in Gruppen zu erfolgen, der Ansturm und das Interesse der Mediziner kannten keine Grenzen. 